\title{
Clarifications to the paper "Holographic characteristics of a 1-mm-thick photopolymer to be used in holographic memories"
}

\author{
Manuel Ortuño, Sergi Gallego, Celia García, Cristian Neipp, and Inmaculada Pascual
}

\begin{abstract}
We have corrected typing errors related to the characterization of the dynamic range of the acrylamide photopolymer described in an earlier study [Appl. Opt. 42, 7008 (2003)]. The $M$ number is expressed as $M$ /\# instead of M\# as appears in the text. The value calculated from the experimental results that are included in the article is $M / \#=3.8$ instead of 38 as appears in the text. (C) 2005 Optical Society of America

OCIS codes: $\quad 090.2900,090.4220,090.7330$.
\end{abstract}

The $M$ number characterizes the dynamic range of a holographic recording medium and enables the holographic data-storage capacity of different types of materials to be compared. It was introduced by Mok et $a l .{ }^{1}$ and was originally expressed as $M / \#$. In our earlier study ${ }^{2}$ it appears as M\#. It is defined by the following expression:

$$
\eta=\left(\frac{M / \#}{M}\right)^{2} .
$$

For a large number of holograms, $M$ is the number of holograms recorded by means of multiplexing and $\eta$ is the equalized diffraction efficiency. From Fig. 8 in our earlier study ${ }^{2}$ it is possible to calculate the experimental value of the dynamic range used to record the nine holograms by the expression

$$
M / \#=\sum_{i=1}^{M} \eta_{i}{ }^{1 / 2}
$$

where $\eta_{i}$ represents the maximum diffraction efficiency reached by each hologram in the reconstruc-

All the authors are with the Universidad de Alicante, Apartado 99, Alicante E-03080, Spain. M. Ortuño, S. Gallego, C. García, and I. Pascual (pascual@ua.es) are with the Departamento Interuniversitario de Óptica. C. Neipp is with the Departamento de Física, Ingeniería de Sistemas y Teoría de la Señal.

Received 1 October 2004; revised manuscript received 29 October 2004; accepted 29 October 2004.

0003-6935/05/081448-01\$15.00/0

(C) 2005 Optical Society of America
Table 1. $\quad \mathbf{D E}_{\max }, \eta_{i}$, and $\eta_{i}^{1 / 2}$ for Each Hologram in Fig. 8 of Ref. 2

\begin{tabular}{cccc}
\hline Hologram & $\mathrm{DE}_{\max }(\%)$ & $\eta_{i}$ & $\eta_{i}{ }^{1 / 2}$ \\
\hline 1 & 15.62 & 0.1562 & 0.3952 \\
2 & 18.13 & 0.1813 & 0.4258 \\
3 & 23.85 & 0.2385 & 0.4884 \\
4 & 20.51 & 0.2051 & 0.4529 \\
5 & 32.16 & 0.3216 & 0.5671 \\
6 & 20.66 & 0.2066 & 0.4545 \\
7 & 21.16 & 0.2116 & 0.4600 \\
8 & 7.29 & 0.0729 & 0.2700 \\
9 & 8.87 & 0.0887 & 0.2978 \\
\hline
\end{tabular}

tion and $M=9$. Table 1 shows the $\mathrm{DE}_{\max }$ values represented in Fig. 8 of Ref. 2 and the corresponding $\eta_{i}, \eta_{i}{ }^{1 / 2}$ for each hologram. From the sum of this last column, we obtain $M / \#=3.8$, a value considered correct by Ihas et al. ${ }^{3}$ This explains the typing error in the paper cited in which it is stated that $M / \#=38$.

To give an example related to the recording that we carried out, to obtain $M / \#=38$, it would be necessary, according to Eq. (2), to record 85 holograms with $\eta_{i}=0.2$ or 170 holograms with $\eta_{i}=0.05$.

\section{References}

1. F. Mok, G. W. Burr, and D. Psaltis, "System metric for holographic memory systems," Opt. Lett. 21, 896-898 (1996).

2. M. Ortuño, S. Gallego, C. García, C. Neipp, and I. Pascual, "Holographic characteristics of a 1-mm-thick photopolymer to be used in holographic memories," Appl. Opt. 42, 7008-7012 (2003).

3. B. C. Ihas, A. J. Hill, and M. Schnoes, "Comment on 'Holographic characteristics of a 1-mm-thick photopolymer to be used in holographic memories'," Appl. Opt. 43, 4017 (2004). 